\title{
Treatment Strategy for Frozen Shoulder
}

\author{
Chul-Hyun Cho, MD, Ki-Choer Bae, MD, Du-Han Kim, MD \\ Department of Orthopedic Surgery, Dongsan Medical Center, Keimyung University School of Medicine, Daegu, Korea
}

Frozen shoulder (FS) is a common shoulder disorder characterized by a gradual increase of pain of spontaneous onset and limitation in range of motion of the glenohumeral joint. The pathophysiology of FS is relatively well understood as a pathological process of synovial inflammation followed by capsular fibrosis, but the cause of FS is still unknown. Treatment modalities for FS include medication, local steroid injection, physiotherapy, hydrodistension, manipulation under anesthesia, arthroscopic capsular release, and open capsular release. Conservative management leads to improvement in most cases. Failure to obtain symptomatic improvement and continued functional disability after 3 to 6 months of conservative treatment are general indications for surgical management. However, there is no consensus as to the most efficacious treatments for this condition. In this review article, we provide an overview of current treatment methods for FS.

Keywords: Frozen shoulder, Adhesive capsulitis, Treatment, Shoulder

Frozen shoulder (FS) is one of the most common, yet challenging clinical disorder presenting to the orthopedic surgeon. It is a disease characterized by a significant decrease of active and passive range of motion (ROM) of the glenohumeral joint along with pain. The prevalence rate of FS is $2 \%-5 \%$, and it occurs more commonly in women. ${ }^{1,2)}$ Along with the increase in the comorbidities and changes in lifestyle, the incidence of FS is increasing. ${ }^{3,4)}$ But, the natural course and pathogenesis of FS have not been widely investigated and are still unknown. According to the research so far, FS can be divided into three phases: freezing (insidious onset of shoulder pain with progressive loss of motion), frozen (gradual subsidence of pain, plateauing of stiffness with equal active and passive ROM), and thawing (gradual improvement of motion and resolution of symptoms). ${ }^{5}$ Macroscopic findings include thickening and congestion of the capsule, with an inflamed appearance, particularly around the rotator interval, of the cora-

Received April 15, 2019; Accepted April 18, 2019

Correspondence to: Du-Han Kim, MD

Department of Orthopedic Surgery, Dongsan Medical Center, Keimyung University School of Medicine, 56 Dalseong-ro, Jung-gu, Daegu 41931, Korea

Tel: +82-53-258-7930, Fax: +82-53-258-4773

E-mail: osmdkdh@dsmc.or.kr cohumeral and middle glenohumeral ligaments (Fig. 1). ${ }^{6}$ Microscopically the affected capsule has a higher number of fibroblasts, mast cells, macrophages, and T cells. This synovitis is associated with the increased fibrotic growth factors, inflammatory cytokines, and interleukins. ${ }^{7,8}$

A primary or idiopathic FS occurs when there is no exogenous cause or preexisting condition or may be associated with another systemic illness. The most common association is diabetes mellitus and the incidence is reported to be $10 \%-36 \% .{ }^{9)}$ Thyroid disease, adrenal disease, cardiopulmonary disease, and hyperlipidemia are also known to be related. ${ }^{9)}$ FS with an identifiable traumatic (fracture, dislocation, and soft tissue injury) or nontraumatic (osteoarthritis, rotator cuff tendinopathy, and calcific tendinitis) shoulder pathology are categorized as secondary FS. ${ }^{10)}$

Traditionally, FS has been regarded as a self-limiting and benign disease with complete recovery of pain and ROM. However, this condition can sometimes last for years. In one study, 50\% of patients were still experiencing pain or stiffness of the shoulder at a mean of 7 years from the onset of the condition, although only $11 \%$ reported functional limitation. ${ }^{11)}$ Reeves ${ }^{5}$ in a prospective study of 41 patients with 5 to 10 years' follow-up, found that only $39 \%$ of patients had full recovery. This long period of pain and disability deprive the patients of their routine life and occupational and recreational activities. Although appropriate treatment is needed for a rapid return to their own 

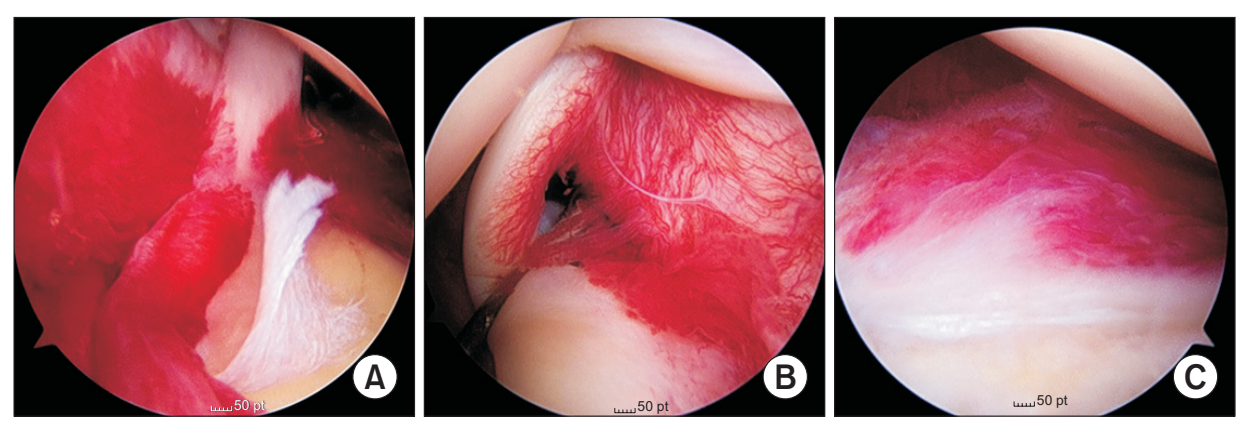

Fig. 1. (A, B) Arthroscopic findings of frozen shoulder. Inflammatory synovitis in the glenohumeral joint. (C) Middle glenohumeral ligament with inflammatory changes.

life, definitive treatment strategies have not been established and many different management strategies are used. In this review article, we provide an overview of treatment methods for FS and discuss proper treatment strategies for FS.

\section{CONSERVATIVE TREATMENT}

Common conservative treatments include oral medication, physical therapy, exercise, steroid injection, and hydrodilatation. These initial conservative managements may be successful in up to $90 \%$ of patients. ${ }^{12)}$ It is important to note the phase being treated because of differences in symptoms at each phase. ${ }^{5,13,14)}$ In freezing phase (duration, 10-36 weeks), pain is most prominent. Steroid injection provides rapid pain relief, mainly in the short-term period. ${ }^{13,14)}$ In frozen phase (4-12 months), pain gradually subsides but restricted ROM is predominant. In this phase, therapy should focus on increasing ROM, such as mobilization techniques or distension for which limited evidence was found. ${ }^{13,14)}$ In the thawing phase (12-42 months), there is minimum pain and progressive improvement in ROM. As pain and muscular inhibition result in compensatory movements of the scapula, the role of adaptation of scapular motion could be important in managing rehabilitation in FS. ${ }^{9,13)}$

\section{Medication}

During the initial painful freezing stages, treatment strategy is directed at pain relief. Although it is traditional to give patients nonsteroidal anti-inflammatory drugs (NSAIDs), NSAIDs alone have no effect on the natural course of FS. ${ }^{13,15)}$ There are no randomized controlled trials that confirm the effectiveness of NSAIDs in the specific condition of FS.

Oral administration of corticosteroid is also used in the treatment of FS. Canbulat et al. ${ }^{16)}$ reported that oral glucocorticoids $(0.5 \mathrm{mg} / \mathrm{kg} /$ day methylprednisolone) in 33 FS patients improved clinical outcomes: the mean visual analog scale (VAS) score, from 6.3 initially to 0.2 at 6-month follow-up; the mean Constant score, from 28.3 initially to 94.8 at first-year follow-up; the mean American Shoulder and Elbow Surgeons score, from 25.9 initially to 98.7 at first-year follow-up. In one randomized clinical trial of 40 patients performed by Lorbach et al., ${ }^{17)}$ patients with idiopathic FS were treated with an oral corticosteroid treatment regimen (20 patients) or intra-articular injection of corticosteroid (20 patients). In the patients treated with the oral regimen, significant improvements were found for pain and functional outcomes at the 4-week follow-up. However, the patients treated with an intra-articular injection showed superior results in objective shoulder scores, ROM, and patient satisfaction compared with the oral steroid group. ${ }^{17)}$ Buchbinder et al. ${ }^{18)}$ reported the results of oral prednisolone for the treatment of FS in a randomized, double-blinded, placebo-controlled study and found significant improvement in the study group at 3 weeks. As described in the previous studies, oral steroid treatment seems to provide early benefit both in terms of pain relief and functional outcomes; however, long-term benefit has not yet been established. One systematic review reported on the use of oral steroid in the treatment of FS (five trials, 179 patients). In three high-quality trials, oral steroids were compared with placebo or observation. No significant differences were found in pain in the short term and in pain and ROM in the long term. ${ }^{19)}$

Calcitonin is a polypeptide hormone secreted from parafollicular cells of the thyroid. Although the mechanism of action of calcitonin is not fully understood, it plays a significant role in managing rheumatoid arthritis, complex regional pain syndrome, fracture, and metastasis of bone tumor. ${ }^{20,21)}$ And it is thought to decrease the systemic inflammatory response and stimulate the release of endorphins. ${ }^{22)}$ A double-blinded randomized clinical trial (level of evidence II) of 64 patients with FS compared intranasal calcitonin and placebo for 6 weeks. Physiotherapy and NSAIDs were administered equally to both groups. At 6 weeks, both groups had significant improvement in pain, ROM, and functional outcomes. However, the im- 
Cho et al. Treatment Strategy for Frozen Shoulder

Clinics in Orthopedic Surgery • Vol. 11, No. 3, $2019 \bullet$ www.ecios.org

provement in the calcitonin group was more notable than that in the placebo group. But there are few well-designed studies; therefore, further research is needed to evaluate whether a calcitonin has an effect on the treatment of FS.

\section{Physiotherapy}

Physiotherapy is widely adopted as an initial treatment in many shoulder conditions including FS. ${ }^{23,24)}$ Physiotherapy should include an exercise program that can restore shoulder motion. The patient should be placed on an exercise program with the goal of regaining and maintaining motion. Patients receiving exercise therapy should begin an active assisted ROM exercise program as well as gentle passive stretching exercises including forward elevation, internal and external rotation, and cross body adduction. These exercises should be performed five to six times per day. And it is important to perform multiple 5- to 10-minute sessions per day as the shoulder will become stiff again in the time between sessions. ${ }^{25)}$

Good results have been reported with physiotherapy itself or in comparison with other conservative management. ${ }^{24,26,27)}$ Russell et al. ${ }^{26)}$ conducted a blinded, randomized, controlled study comparing the efficacy of three treatment regimens: exercise class plus home exercises, individual multimodal physiotherapy plus home exercises, and home exercises alone. They found the exercise class group showed significant improvement in Oxford and Constant scores. The improvement in ROM was significantly greater in the physiotherapy group than the exercise alone groups. They emphasized that compared with exercise, physiotherapy interventions lead to significant improvement in anxiety, which is strongly correlated with symptoms. Griggs et al. ${ }^{24)}$ reported that $90 \%$ of the 75 patients treated with use of a specific four-direction shoulder-stretching exercise program obtained satisfactory results at a mean follow-up of 22 months. Sun et al. ${ }^{27)}$ undertook a systematic review and meta-analysis of randomized controlled trails to evaluate the effect of steroid injection and physiotherapy. They concluded that both interventions had similar effect on improving shoulder function, increasing passive motion, and decreasing pain in FS.

Many studies have demonstrated physiotherapy as an adjunctive intervention that provides good results. NSAIDs were proven to be more effective when used in combination with physiotherapy as compared to NSAIDs alone. ${ }^{28)}$ Similarly, steroid injection used in combination with physiotherapy resulted in better outcomes compared to injection alone. . $^{29,30)}$

\section{Corticosteroid Injection}

Although injection of corticosteroids is an invasive procedure and associated with risks such as septic arthritis, ${ }^{17)}$ it is useful to reduce pain and disability of the patients during painful or freezing stages. ${ }^{3,31)}$ There have been numerous randomized studies that examined the early efficacy of steroid injection. ${ }^{32-34)}$ In a double-blinded, sham-controlled randomized clinical trial, ultrasonography-guided intraarticular and rotator interval steroid injections in 122 patients with FS resulted in a notable decrease in pain at 6 weeks. The result was maintained at 12 weeks, but not at 26 weeks. And there was no difference between the group who received intra-articular injection and the group who received both intra-articular and rotator interval injections. ${ }^{35)}$ Ryans et al. ${ }^{36)}$ reported results of a four-way randomized controlled trial comparing (1) an injection of triamcinolone plus physiotherapy, (2) injection alone, (3) placebo injection plus physiotherapy, and (4) placebo injection alone. At 6 weeks' follow-up, corticosteroid injection groups were significantly improved in terms of shoulder-related disability, and physiotherapy groups had improvement in ROM. However, all groups had improved to a similar degree with respect to all outcome measures at 16 weeks. In 2011, Griesser et al. ${ }^{33)}$ conducted a systematic review of existing level I and II evidence studies about intra-articular injection for FS. Eight studies comprising 409 shoulders met their criteria for inclusion. Even though the mean modified Coleman methodology score of the included studies was as low as 44 , all treatments resulted in improved clinical outcome with a trend toward greater improvement in 36-Item Short Form Survey (SF-36) scores in association with steroid injection as compared with manipulation under anesthesia (MUA).

There is no clear evidence as to which injection site is most effective. In a study by Oh et al., ${ }^{37)}$ a glenohumeral joint steroid injection (37 shoulders) was not superior to a subacromial injection (34 shoulders) for patients with primary FS at 6 and 12 weeks, even though the glenohumeral injection led to earlier pain relief compared with the subacromial injection. For ROM, no statistical differences were found between the groups at any of the follow-up assessments. Shin and Lee ${ }^{38)}$ randomly divided 191 patients into four groups who underwent one of the following treatment methods: corticosteroid injection into the subacromial space, glenohumeral joint, or glenohumeral joint combined with subacromial space, or oral NSAID medication. They found that steroid injection provided faster pain relief, a higher level of patient satisfaction, and earlier recovery in shoulder function than medication. But, the efficacy of a corticosteroid injection was not found to be 
related to the site of injection. Cho et al. ${ }^{39)}$ randomly assigned 126 patients with idiopathic FS to intra-articular injection group, subacromial injection group, or combination (intra-articular plus subacromial) injection group. Their results revealed that intra-articular injection or combination injection was superior to subacromial injection. And combination injections showed an additive effect on internal rotation angle.

\section{Hydrodistension}

Andren and Lundberg ${ }^{40)}$ first described hydrodistension (HD) in 1965 to treat the adhesive glenohumeral joint by expansion of the capsule. Although therapeutic regimens will differ, this technique is the installation of a large volume of saline, steroid, local anesthetic, and contrast agent into the glenohumeral joint under imaging guidance. ${ }^{41-43)}$ Most studies comment on a procedure to achieve capsular rupture but have not investigated this. ${ }^{41,42)}$ There is no evidence to determine whether capsule rupture must be achieved or whether capsular distension is most important.

HD has been reported to provide short-term benefits regarding pain, ROM, and function in FS. ${ }^{41)}$ Haughton et $\mathrm{al}^{42)}$ reported results of $\mathrm{HD}$ in 76 patients with a mean follow-up of 3.5 months. The Oxford shoulder score improved from a mean of 20.6 preoperatively to a mean of 32.7 postoperatively. A Cochrane review in 2008 demonstrated only silver level evidence to support $\mathrm{HD}$ as a treatment modality for short-term improvement of pain, ROM, and function. ${ }^{41)}$

Good long-term outcome was also reported in several studies. ${ }^{43,44)}$ Watson et al. ${ }^{44)}$ demonstrated the efficacy of HD in 41 patients with a 2-year follow-up. Primary outcomes included Shoulder Pain and Disability Index and Shoulder Disability Index and secondary included ROM. They found significant improvement in all outcomes over the follow-up period, and these benefits associated with $\mathrm{HD}$ and physiotherapy continued to improve or were maintained in the long term, up to 2 years after this procedure. Clement et al. ${ }^{43)}$ also demonstrated similar results of arthrographic distension in 53 FS with a mean follow-up of 14 months. The Oxford shoulder score improved from a mean of 22.3 at baseline to a mean of 39.2 at final followup and VAS score decreased from a mean initial value of 7.1 to a mean of 3.6. In 2018, Saltychev et al. ${ }^{45)}$ evaluated the evidence on the effectiveness of HD in treatment of FS in a meta-analysis. The seven included studies assessed the essential effect of HD combined with corticosteroid versus corticosteroid alone. They reported that the amount of injected solution did not have a substantial effect on pain or
ROM and concluded that HD has only a small, clinically insignificant effect when treating FS. ${ }^{45)}$

\section{SURGICAL PROCEDURE}

Despite the self-limited natural history of the disease, some patients fail to achieve desired outcomes with nonoperative management. ${ }^{9,22,46)}$ Factors that influence the decision on surgical management include severity and duration of symptoms as well as response to conservative treatment. $^{3,22)}$

General indications for surgery are persistent pain and limited motion despite a minimum 3 to 6 months of nonoperative management including medication, local injections, or physiotherapy. ${ }^{9,46,47}$ Levine et al. ${ }^{12)}$ reported that patients with more severe initial symptoms, younger age at the time of onset, and reduction in motion despite 4 months of compliance with therapy are most likely to require surgery.

As with the increase in patients with FS, surgical intervention for FS is common these days. The overall incidence of FS surgery was calculated as 2.67 procedures per 10,000 general population per year and as 7.55 for those aged 40-60 years. ${ }^{48,49)}$ Management of FS amongst doctors varies substantially and is highly based on personal experience and training rather than published evidence. ${ }^{49)} \mathrm{Op}$ erative treatment methods include MUA and arthroscopic or open capsular release. As arthroscopic capsular release (ACR) is a reliable treatment option with many advantages over open surgery, the indications of open release have decreased and open release is now rarely performed.

\section{Manipulation under Anesthesia}

MUA involves passive tearing of the thickened inflamed capsule and contracted ligaments (Fig. 2). It is mainly performed under general anesthesia; however, recent development of ultrasound technology enabled it with brachial
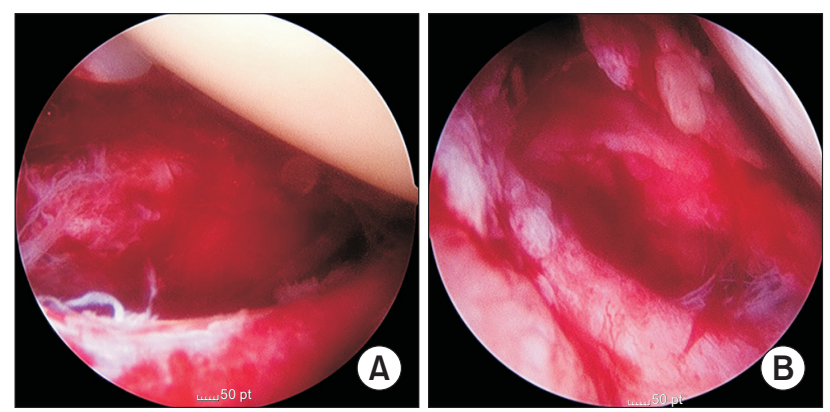

Fig. 2. (A, B) Capsular tearing after manipulation under anesthesia. 
plexus or cervical nerve root block. ${ }^{50,51)}$ Magnetic resonance imaging after MUA shows capsular tears (midsubstance and humeral avulsion of glenohumeral ligaments), labral tears, or bone bruises of the humeral head. ${ }^{51)}$ And arthroscopic findings of post-MUA include hemarthrosis, tearing of the joint capsule or rotator cuff, superior labrum from anterior to posterior tear lesion, labral tear, middle glenohumeral ligament rupture. ${ }^{52)}$

Even though the optimal timing of MUA has not yet been determined, Vastamaki et al. ${ }^{53)}$ suggested that if conservative management failed, the best time for MUA might be between 6 and 9 months from the onset of the symptom. They believed that too early manipulation (before 6 months after the onset of symptom) may lead to a recurrence because the disease is still at the inflammation stage. ${ }^{53)}$ MUA has been used extensively with satisfactory short- and long-term results. Thomas et al..$^{54)}$ noted that 246 patients with an idiopathic FS treated by MUA had good clinical outcomes at a mean of 42 months. Vastamaki et al. ${ }^{55)}$ evaluated 26 patients after MUA for FS and a substantial increase in ROM and pain relief were found at 7 years' follow-up. These authors also showed maintained improvement in ROM, pain, and function at 23 years in a group of 16 shoulders treated with MUA. ${ }^{55)}$

However, the results of MUA, when compared to HD or steroid injection, are equivocal at best. ${ }^{56)}$ Quraishi et al. ${ }^{57)}$ performed HD in 20 shoulders with FS and compared the results with those of manipulation performed in 18 shoulders. Most of their patients were treated successfully. However, the Constant scores in the HD group were significantly better than those in the manipulation group over the 6 months of follow-up. Furthermore, 94\% of patients were very satisfied or satisfied after HD, compared with $81 \%$ of manipulation group at the final follow-up. ${ }^{57)}$

The recurrence rate after MUA varies from 3\% to $40 \%{ }^{58-60)}$ Jenkins et al. ${ }^{59)}$ reported that $36 \%$ of patients who had diabetic FS required a repeat MUA versus $15 \%$ for nondiabetic shoulders. With the repeat MUA, $85 \%$ of the patients were successfully treated. ${ }^{59)}$ Woods and Loganathan $^{58)}$ performed MUA in 730 patients with FS. A further MUA was undertaken in $17.8 \%$ and patients with type-1 diabetes mellitus were at $38 \%$ increased risk of requiring a further MUA.

Surgeons should be always concerned about the risk of complications related to MUA, including humeral shaft fracture, glenoid fracture, rotator cuff tear, dislocation of shoulder, and traction injury to nerve. ${ }^{61,62)}$ Tsvieli et al. ${ }^{63)}$ reported that understanding of Codman's paradox enabled them to minimize the risk of complications during the MUA. This paradox leads to an apparent $180^{\circ}$ rotation in the shoulder during two sequential axial movements. ${ }^{63)}$ The first step of their MUA method is elevation of the arm in the plane of the scapula that is stabilized with a very short lever arm. The second step is bringing the arm that is in full external rotation down by the side without any rotation. In this setting, MUA can be performed without any rotation torque on the humerus. ${ }^{63)}$

\section{Arthroscopic Capsular Release}

Due to complications of MUA and advances in arthroscopic techniques, ACR has become the most frequently used surgical intervention that was previously shown to confer lasting long-term improvements in symptoms (Fig. 3). ${ }^{49}$ ACR also allows for visual confirmation of the diagnosis as well as the ability to treat concomitant intra-articular and subacromial disease that may be contributing to the primary cause of the problem. ${ }^{25}$

Recently, many studies have shown excellent results both in terms of pain relief and ROM gain with ACR. In a study by Le Lievre and Murrell, ${ }^{64)} 49$ shoulders treated with an ACR obtained early significant improvements in ROM, pain relief, and function. These improvements were maintained at 7 years. ${ }^{64)}$ Furthermore, even when compared with other procedures such as HD and MUA, ACR had good clinical results. Gallacher et al. ${ }^{65)}$ compared the 6-month follow-up results of HD (20 patients) for FS with ACR (19 patients). They reported that patients randomized to ACR showed a significantly higher Oxford shoulder score at 6 months than the HD group. ${ }^{65)}$

There is a wide variation in the way ACR is carried out, ranging from partial release to a full $360^{\circ}$ release. Also there are various debates in the literature regarding the extent of release. Several authors recommended release of the posterior capsule, and it was believed to have advantages regarding the recovery of internal rotation. ${ }^{6-70)}$ On the contrary, Chen et al. ${ }^{71)}$ reported that although the

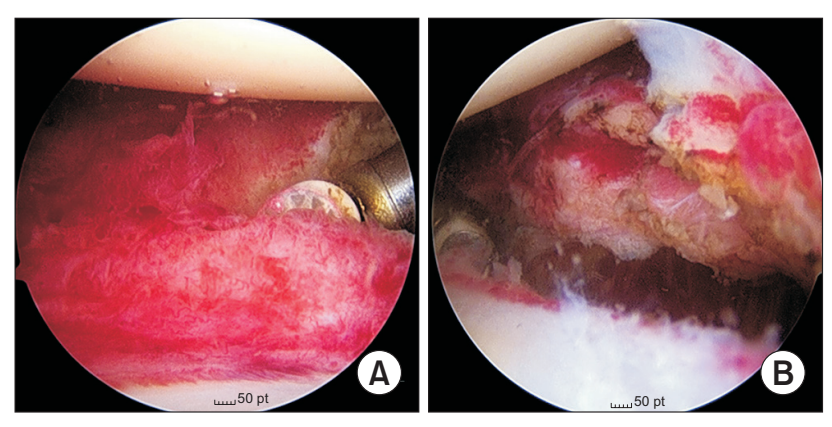

Fig. 3. (A) Intraoperative arthroscopic image showing a release of the anterior capsule with an ablation device. (B) The appearance of the capsule after radiofrequency ablation. 
ROM (abduction and internal rotation) improvement was more significant in the addition of posterior release within the first 3 months after ACR, there was no significant improvement in function or internal rotation with the addition of posterior release at mean of 28 months after surgery.

Some surgeons prefer MUA followed by ACR, which has also provided satisfactory results. De Carli et al. $^{72)}$ followed up 23 patients who underwent MUA and arthroscopic arthrolysis for a minimum of 12 months and compared the results with those of intra-articular steroid injection performed in 21 patients. They found patients of MUA followed by arthroscopic surgery accomplished their goal by the 6-week follow-up, whereas in the injection group, the same result was obtained at 12 weeks. Grant et al. $^{73)}$ conducted a systematic review of 22 studies that compare outcomes between MUA, capsular release, or a combination of both. Of the study participants, the median age was 52 years and $60 \%$ were women. They concluded that even though the quality of evidence available was low, there was little benefit of ACR instead of or in addition to MUA.

Overall, ACR in FS is a safe procedure with a low complication (nerve injury, chondrolysis, or instability) rate; however, caution to axillary nerve injury is needed. To prevent possible injury to the axillary nerve, some authors were very cautious about the inferior release. ${ }^{71)}$ In cadaveric dissections, the teres minor branch of axillary nerve was the closest to the 5:30 and 6 oclock position on the inferior glenoid rim. At this position, the average distance between the axillary nerve and the glenoid rim was $12.4 \mathrm{~mm}$ (10 to $25 \mathrm{~mm}$ ) and the nerve lay at an average of $2.5 \mathrm{~mm}$ from the inferior glenohumeral ligament. The abduction-neutral position resulted in the greatest distance between the inferior glenoid and the axillary nerve. ${ }^{74,75)}$

\section{Rehabilitation}

Postoperative rehabilitation is another very important point in the surgical treatment. If possible, rehabilitation should be started as soon as possible in order to maintain movement gain achieved intraoperatively. Some authors recommended the use of continuous interscalene catheter for anesthetic infusion in the early postoperative period to improve pain relief and patient satisfaction. ${ }^{76)}$ An arm sling is only used for comfort for a few days after surgery and must not be continued by 1 week. Patients are encouraged not to sleep in the arm sling. A home stretching program is taught and should be done three times per day. ${ }^{22,47)}$

\section{CONCLUSIONS}

FS, commonly encountered in general orthopedic practice, is a condition of pain and stiffness with consequent functional impairment. Appropriate treatment decisions for FS require a comprehensive understanding of pathophysiology, patient's systemic medical condition, functional demands, severity of symptoms, and response for nonoperative treatment. The majority will experience resolution when treated conservatively; thus, conservative management should be the first option.

NSAIDs may relieve pain and reduce sleep disturbance, but they do not have a substantial effect on recovery. Oral steroid may provide rapid pain relief and ROM recovery in the short term. Physiotherapy is so widely accepted that it should be used in the conservative management of FS. When patients have the most pain, steroid injections can be beneficial in the early period of the disease (particularly, in the first 6 weeks). But longer-term results would show no difference between patients treated with steroids and control subjects. There is still debate on the appropriate steroid injection site. The HD alone appears to provide only a small, clinical benefit, and there is no evidence to suggest any superiority to other treatments.

Initial conservative management may be successful in up to $90 \%$ of patients. Patients who are regressing despite appropriate therapy are likely to require surgical intervention. MUA has been used extensively with satisfactory outcomes. However, surgeons always need to take caution to avoid iatrogenic complications and should explain the possibility of recurrence in patients with diabetes. Although the extent of additional capsule that should be released remains controversial, ACR is a reliable treatment method, with a low complication rate, for restoring function and reducing pain in patients with FS. Patients should begin progressive ROM exercises as soon as possible under the supervision of a trained therapist.

\section{CONFLICT OF INTEREST}

No potential conflict of interest relevant to this article was reported. 


\section{REFERENCES}

1. Robinson PM, Norris J, Roberts CP. Randomized controlled trial of supervised physiotherapy versus a home exercise program after hydrodilatation for the management of primary frozen shoulder. J Shoulder Elbow Surg. 2017;26(5):757-65.

2. Lee SY, Lee KJ, Kim W, Chung SG. Relationships between capsular stiffness and clinical features in adhesive capsulitis of the shoulder. PM R. 2015;7(12):1226-34.

3. Brue S, Valentin A, Forssblad M, Werner S, Mikkelsen C, Cerulli G. Idiopathic adhesive capsulitis of the shoulder: a review. Knee Surg Sports Traumatol Arthrosc. 2007;15(8):1048-54.

4. Ogilvie-Harris DJ, Biggs DJ, Fitsialos DP, MacKay M. The resistant frozen shoulder: manipulation versus arthroscopic release. Clin Orthop Relat Res. 1995;(319):238-48.

5. Reeves B. The natural history of the frozen shoulder syndrome. Scand J Rheumatol. 1975;4(4):193-6.

6. Whelton C, Peach CA. Review of diabetic frozen shoulder. Eur J Orthop Surg Traumatol. 2018;28(3):363-71.

7. Kabbabe B, Ramkumar S, Richardson M. Cytogenetic analysis of the pathology of frozen shoulder. Int J Shoulder Surg. 2010;4(3):75-8.

8. Tamai K, Akutsu M, Yano Y. Primary frozen shoulder: brief review of pathology and imaging abnormalities. J Orthop Sci. 2014;19(1):1-5.

9. Dias R, Cutts S, Massoud S. Frozen shoulder. BMJ. 2005;331(7530):1453-6.

10. Robinson CM, Seah KT, Chee YH, Hindle P, Murray IR. Frozen shoulder. J Bone Joint Surg Br. 2012;94(1):1-9.

11. Shaffer B, Tibone JE, Kerlan RK. Frozen shoulder: a longterm follow-up. J Bone Joint Surg Am. 1992;74(5):738-46.

12. Levine WN, Kashyap CP, Bak SF, Ahmad CS, Blaine TA, Bigliani LU. Nonoperative management of idiopathic adhesive capsulitis. J Shoulder Elbow Surg. 2007;16(5):569-73.

13. Favejee MM, Huisstede BM, Koes BW. Frozen shoulder: the effectiveness of conservative and surgical interventions-systematic review. Br J Sports Med. 2011;45(1):49-56.

14. Hannafin JA, Chiaia TA. Adhesive capsulitis: a treatment approach. Clin Orthop Relat Res. 2000;(372):95-109.

15. Bulgen DY, Binder AI, Hazleman BL, Dutton J, Roberts S. Frozen shoulder: prospective clinical study with an evaluation of three treatment regimens. Ann Rheum Dis. 1984;43(3):353-60.

16. Canbulat N, Eren I, Atalar AC, Demirhan M, Eren SM, Ucak A. Nonoperative treatment of frozen shoulder: oral glucocorticoids. Int Orthop. 2015;39(2):249-54.

17. Lorbach O, Anagnostakos K, Scherf C, Seil R, Kohn D, Pape D. Nonoperative management of adhesive capsulitis of the shoulder: oral cortisone application versus intra-articular cortisone injections. J Shoulder Elbow Surg. 2010;19(2):1729.

18. Buchbinder R, Hoving JL, Green S, Hall S, Forbes A, Nash P. Short course prednisolone for adhesive capsulitis (frozen shoulder or stiff painful shoulder): a randomised, double blind, placebo controlled trial. Ann Rheum Dis. 2004;63(11):1460-9.

19. Buchbinder R, Green S, Youd JM, Johnston RV. Oral steroids for adhesive capsulitis. Cochrane Database Syst Rev. 2006;(4):CD006189.

20. Sahin F, Yilmaz F, Kotevoglu N, Kuran B. Efficacy of salmon calcitonin in complex regional pain syndrome (type 1) in addition to physical therapy. Clin Rheumatol. 2006;25(2):143-8.

21. Waldburger M, Meier JL, Gobelet C. The frozen shoulder: diagnosis and treatment. Prospective study of 50 cases of adhesive capsulitis. Clin Rheumatol. 1992;11(3):364-8.

22. Redler LH, Dennis ER. Treatment of adhesive capsulitis of the shoulder. J Am Acad Orthop Surg. 2019;27(12):e544-54.

23. Diercks RL, Stevens M. Gentle thawing of the frozen shoulder: a prospective study of supervised neglect versus intensive physical therapy in seventy-seven patients with frozen shoulder syndrome followed up for two years. J Shoulder Elbow Surg. 2004;13(5):499-502.

24. Griggs SM, Ahn A, Green A. Idiopathic adhesive capsulitis: a prospective functional outcome study of nonoperative treatment. J Bone Joint Surg Am. 2000;82(10):1398-407.

25. Iannotti JP, Williams GR. Disorders of the shoulder: diagnosis and management. 2nd ed. Philadelphia, PA: Lippincott Williams \& Wilkins; 2007.

26. Russell S, Jariwala A, Conlon R, Selfe J, Richards J, Walton M. A blinded, randomized, controlled trial assessing conservative management strategies for frozen shoulder. J Shoulder Elbow Surg. 2014;23(4):500-7.

27. Sun Y, Lu S, Zhang P, Wang Z, Chen J. Steroid injection versus physiotherapy for patients with adhesive capsulitis of the shoulder: a PRIMSA systematic review and meta-analysis of randomized controlled trials. Medicine (Baltimore). 2016;95(20):e3469.

28. Dudkiewicz I, Oran A, Salai M, Palti R, Pritsch M. Idiopathic adhesive capsulitis: long-term results of conservative treatment. Isr Med Assoc J. 2004;6(9):524-6. 
29. Page MJ, Green S, Kramer S, et al. Manual therapy and exercise for adhesive capsulitis (frozen shoulder). Cochrane Database Syst Rev. 2014;(8):CD011275.

30. Chan HB, Pua PY, How CH. Physical therapy in the management of frozen shoulder. Singapore Med J. 2017;58(12):685-9.

31. Song A, Higgins LD, Newman J, Jain NB. Glenohumeral corticosteroid injections in adhesive capsulitis: a systematic search and review. PM R. 2014;6(12):1143-56.

32. Carette S, Moffet H, Tardif J, et al. Intraarticular corticosteroids, supervised physiotherapy, or a combination of the two in the treatment of adhesive capsulitis of the shoulder: a placebo-controlled trial. Arthritis Rheum. 2003;48(3):82938.

33. Griesser MJ, Harris JD, Campbell JE, Jones GL. Adhesive capsulitis of the shoulder: a systematic review of the effectiveness of intra-articular corticosteroid injections. J Bone Joint Surg Am. 2011;93(18):1727-33.

34. Maund E, Craig D, Suekarran S, et al. Management of frozen shoulder: a systematic review and cost-effectiveness analysis. Health Technol Assess. 2012;16(11):1-264.

35. Prestgaard T, Wormgoor ME, Haugen S, Harstad H, Mowinckel P, Brox JI. Ultrasound-guided intra-articular and rotator interval corticosteroid injections in adhesive capsulitis of the shoulder: a double-blind, sham-controlled randomized study. Pain. 2015;156(9):1683-91.

36. Ryans I, Montgomery A, Galway R, Kernohan WG, McKane R. A randomized controlled trial of intra-articular triamcinolone and/or physiotherapy in shoulder capsulitis. Rheumatology (Oxford). 2005;44(4):529-35.

37. Oh JH, Oh CH, Choi JA, Kim SH, Kim JH, Yoon JP. Comparison of glenohumeral and subacromial steroid injection in primary frozen shoulder: a prospective, randomized short-term comparison study. J Shoulder Elbow Surg. 2011;20(7):1034-40.

38. Shin SJ, Lee SY. Efficacies of corticosteroid injection at different sites of the shoulder for the treatment of adhesive capsulitis. J Shoulder Elbow Surg. 2013;22(4):521-7.

39. Cho CH, Kim du H, Bae KC, Lee D, Kim K. Proper site of corticosteroid injection for the treatment of idiopathic frozen shoulder: Results from a randomized trial. Joint Bone Spine. 2016;83(3):324-9.

40. Andren L, Lundberg BJ. Treatment of rigid shoulders by joint distension during arthrography. Acta Orthop Scand. 1965;36:45-53.

41. Buchbinder R, Green S, Youd JM, Johnston RV, Cumpston M. Arthrographic distension for adhesive capsulitis (frozen shoulder). Cochrane Database Syst Rev. 2008;(1):CD007005.
42. Haughton DN, Barton S, Meenan E, et al. Can we improve the outcome of hydrodilatation for adhesive capsulitis? Shoulder Elbow. 2018;10(2):93-8.

43. Clement RG, Ray AG, Davidson C, Robinson CM, Perks FJ. Frozen shoulder: long-term outcome following arthrographic distension. Acta Orthop Belg. 2013;79(4):368-74.

44. Watson L, Bialocerkowski A, Dalziel R, Balster S, Burke F, Finch C. Hydrodilatation (distension arthrography): a long-term clinical outcome series. Br J Sports Med. 2007;41(3):167-73.

45. Saltychev M, Laimi K, Virolainen P, Fredericson M. Effectiveness of hydrodilatation in adhesive capsulitis of shoulder: a systematic review and meta-analysis. Scand J Surg. 2018;107(4):285-93.

46. Itoi E, Arce G, Bain GI, et al. Shoulder stiffness: current concepts and concerns. Arthroscopy. 2016;32(7):1402-14.

47. Neviaser AS, Neviaser RJ. Adhesive capsulitis of the shoulder. J Am Acad Orthop Surg. 2011;19(9):536-42.

48. Kwaees TA, Charalambous CP. Rates of surgery for frozen shoulder: an experience in England. Muscles Ligaments Tendons J. 2016;5(4):276-9.

49. Kwaees TA, Charalambous CP. Surgical and non-surgical treatment of frozen shoulder: survey on surgeons treatment preferences. Muscles Ligaments Tendons J. 2015;4(4):420-4.

50. Ando A, Hamada J, Hagiwara Y, Sekiguchi T, Koide M, Itoi E. Short-term clinical results of manipulation under ultrasound-guided brachial plexus block in patients with idiopathic frozen shoulder and diabetic secondary frozen shoulder. Open Orthop J. 2018;12:99-104.

51. Sasanuma H, Sugimoto H, Kanaya Y, et al. Magnetic resonance imaging and short-term clinical results of severe frozen shoulder treated with manipulation under ultrasoundguided cervical nerve root block. J Shoulder Elbow Surg. 2016;25(1):e13-20.

52. Loew M, Heichel TO, Lehner B. Intraarticular lesions in primary frozen shoulder after manipulation under general anesthesia. J Shoulder Elbow Surg. 2005;14(1):16-21.

53. Vastamaki H, Varjonen L, Vastamaki M. Optimal time for manipulation of frozen shoulder may be between 6 and 9 months. Scand J Surg. 2015;104(4):260-6.

54. Thomas WJ, Jenkins EF, Owen JM, et al. Treatment of frozen shoulder by manipulation under anaesthetic and injection: does the timing of treatment affect the outcome? J Bone Joint Surg Br. 2011;93(10):1377-81.

55. Vastamaki H, Kettunen J, Vastamaki M. The natural history of idiopathic frozen shoulder: a 2- to 27-year followup study. Clin Orthop Relat Res. 2012;470(4):1133-43. 
56. Uppal HS, Evans JP, Smith C. Frozen shoulder: a systematic review of therapeutic options. World J Orthop. 2015;6(2):263-8.

57. Quraishi NA, Johnston P, Bayer J, Crowe M, Chakrabarti AJ. Thawing the frozen shoulder: a randomised trial comparing manipulation under anaesthesia with hydrodilatation. J Bone Joint Surg Br. 2007;89(9):1197-200.

58. Woods DA, Loganathan K. Recurrence of frozen shoulder after manipulation under anaesthetic (MUA): the results of repeating the MUA. Bone Joint J. 2017;99(6):812-7.

59. Jenkins EF, Thomas WJ, Corcoran JP, et al. The outcome of manipulation under general anesthesia for the management of frozen shoulder in patients with diabetes mellitus. J Shoulder Elbow Surg. 2012;21(11):1492-8.

60. Theodorides AA, Owen JM, Sayers AE, Woods DA. Factors affecting short- and long-term outcomes of manipulation under anaesthesia in patients with adhesive capsulitis of the shoulder. Shoulder Elbow. 2014;6(4):245-56.

61. Hamdan TA, Al-Essa KA. Manipulation under anaesthesia for the treatment of frozen shoulder. Int Orthop. 2003;27(2):107-9.

62. Magnussen RA, Taylor DC. Glenoid fracture during manipulation under anesthesia for adhesive capsulitis: a case report. J Shoulder Elbow Surg. 2011;20(3):e23-6.

63. Tsvieli O, Atoun E, Consigliere P, et al. Manipulation under anaesthetic for frozen shoulder using Codman's paradox: a safe and early return of function. Int Orthop. 2018;42(2):339-44.

64. Le Lievre HM, Murrell GA. Long-term outcomes after arthroscopic capsular release for idiopathic adhesive capsulitis. J Bone Joint Surg Am. 2012;94(13):1208-16.

65. Gallacher S, Beazley JC, Evans J, et al. A randomized controlled trial of arthroscopic capsular release versus hydrodilatation in the treatment of primary frozen shoulder. J Shoulder Elbow Surg. 2018;27(8):1401-6.

66. Nicholson GP. Arthroscopic capsular release for stiff shoulders: effect of etiology on outcomes. Arthroscopy. 2003;19(1):40-9.

67. Jerosch J. 360 degrees arthroscopic capsular release in patients with adhesive capsulitis of the glenohumeral jointindication, surgical technique, results. Knee Surg Sports Traumatol Arthrosc. 2001;9(3):178-86.

68. Pearsall AW 4th, Osbahr DC, Speer KP. An arthroscopic technique for treating patients with frozen shoulder. Arthroscopy. 1999;15(1):2-11.

69. Warner JJ, Allen AA, Marks PH, Wong P. Arthroscopic release of postoperative capsular contracture of the shoulder. J Bone Joint Surg Am. 1997;79(8):1151-8.

70. Snow M, Boutros I, Funk L. Posterior arthroscopic capsular release in frozen shoulder. Arthroscopy. 2009;25(1):19-23.

71. Chen J, Chen S, Li Y, Hua Y, Li H. Is the extended release of the inferior glenohumeral ligament necessary for frozen shoulder? Arthroscopy. 2010;26(4):529-35.

72. De Carli A, Vadala A, Perugia D, et al. Shoulder adhesive capsulitis: manipulation and arthroscopic arthrolysis or intra-articular steroid injections? Int Orthop. 2012;36(1):1016.

73. Grant JA, Schroeder N, Miller BS, Carpenter JE. Comparison of manipulation and arthroscopic capsular release for adhesive capsulitis: a systematic review. J Shoulder Elbow Surg. 2013;22(8):1135-45.

74. Price MR, Tillett ED, Acland RD, Nettleton GS. Determining the relationship of the axillary nerve to the shoulder joint capsule from an arthroscopic perspective. J Bone Joint Surg Am. 2004;86(10):2135-42.

75. Zanotti RM, Kuhn JE. Arthroscopic capsular release for the stiff shoulder: description of technique and anatomic considerations. Am J Sports Med. 1997;25(3):294-8.

76. Mariano ER, Afra R, Loland VJ, et al. Continuous interscalene brachial plexus block via an ultrasound-guided posterior approach: a randomized, triple-masked, placebocontrolled study. Anesth Analg. 2009;108(5):1688-94. 